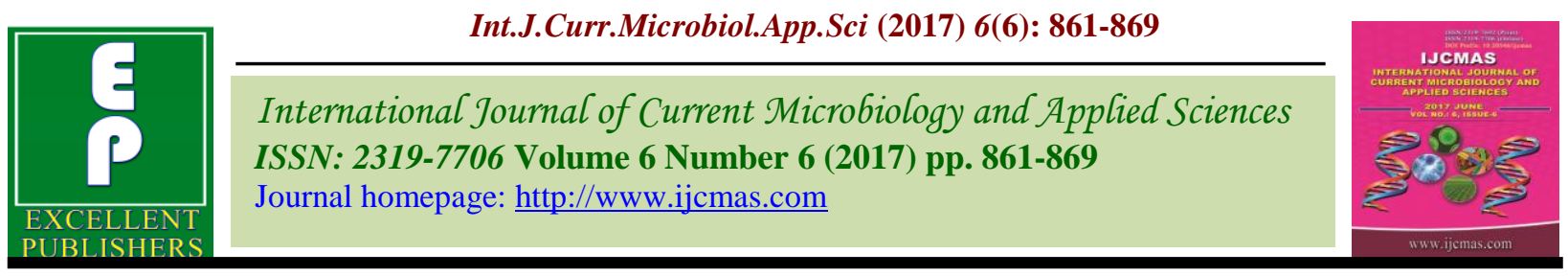

Original Research Article

https://doi.org/10.20546/ijcmas.2017.606.101

\title{
Effect of Different Nitrogen Levels and Varietal Performance on Growth and Yield of Summer Pearlmillet
}

\author{
Neha, Gautam Ghosh, Preeti Choudhary and Shobha Kumari
}

Department of Agronomy, Sam Higginbottom Institute of Agriculture, Technology and Sciences

(Formerly Allahabad Agricultural Institute), (Deemed to-be-University),

Allahabad - 211007 (U.P), India

*Corresponding author

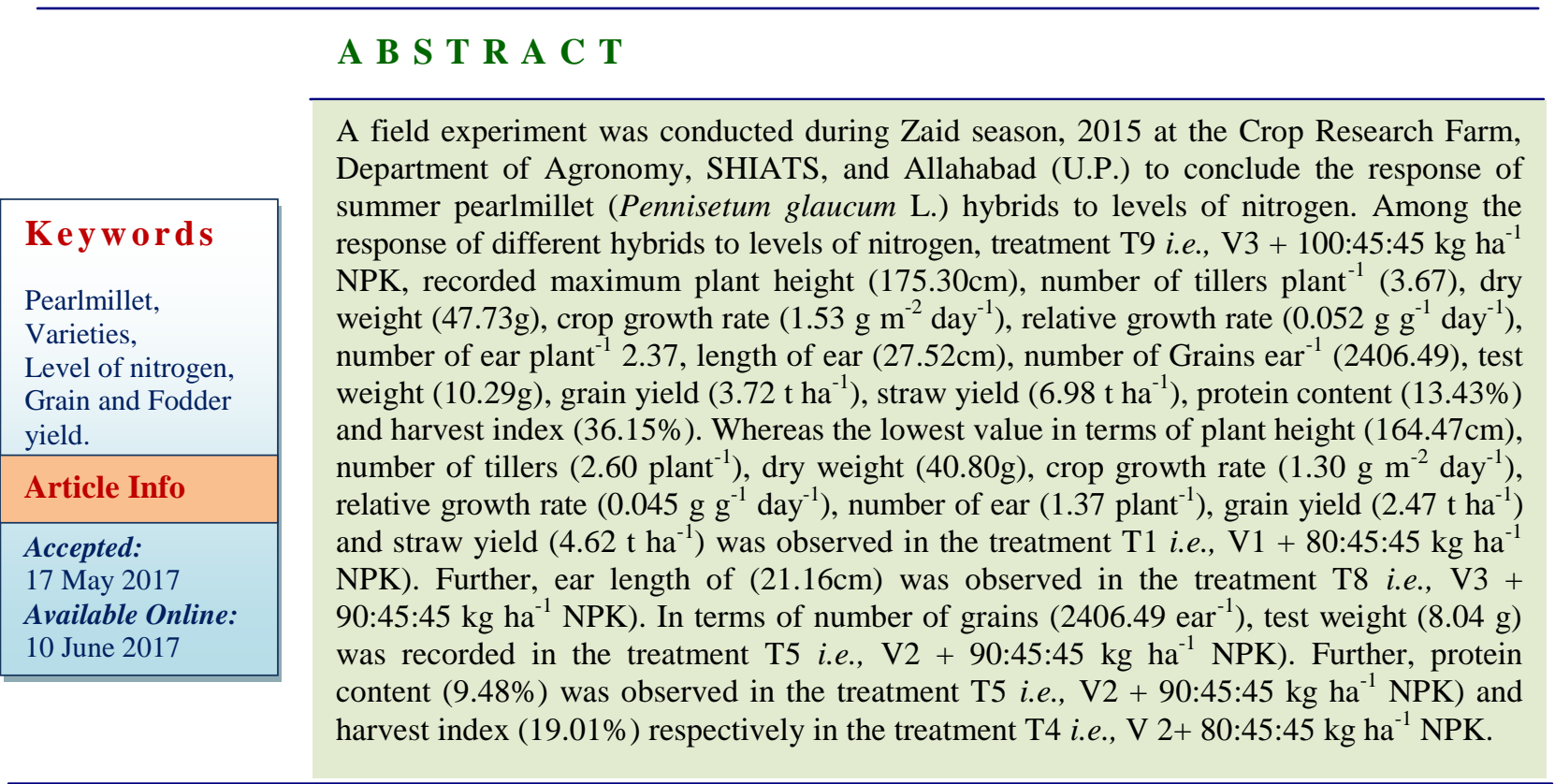

\section{Introduction}

Pearlmillet [Pennisetum glaucum (L.)] is largely grown for grain and fodder purpose under those situations where other crops generally fail. Pearlmillet as a food crop is limited to the developing countries in Asia, and particularly in Africa and ranked sixth in the world following rice, wheat, corn, barley and sorghum (Anonymous, 2010-11). The important pearlmillet growing countries are India, China, Nigeria, Pakistan, Sudan, Egypt, Arabia, and Russia. It is estimated that over 95\% of pearlmillet production is used as food, the reminder being divided between animal and poultry feed (7\%), other uses (seed, bakery products, snacks, etc.) and waste. Pearlmillet is used in flat breads (roti) or mixed up to $25 \%$ with wheat flour for use in yeast breads. The genus Pennisetum is distributed throughout the tropics and subtropics of the world. It includes about 140 species, one African species $P$. glaucum (L.) R. Br. Emend Stuntz was domesticated as the cereal pearlmillet, and another African species, P. Purpureum schumach became widely distributed as a tropical forage grass. Pearlmillet is the most drought tolerant in all 
domesticated cereals, and soon after its domestication it became widely distributed across the semi-arid tropics of Africa and Asia.

Pearlmillet is grown where no other cereal will yield grain, in regions with $200-800 \mathrm{~mm}$ of annual rainfall. In India, pearlmillet is popularly known as Bajra, and it is the fourth most important cereal crop after rice, wheat and sorghum. It has the greatest potential among all the millets. Annual planting area of the country under pearlmillet is 9.5 million hectares producing nearly 10.1 million tonnes of grains with productivity of $10.44 \mathrm{q} \mathrm{ha}^{-1}$ (Economic Survey of India, 2011).

The major producing states are Rajasthan (46\%), Maharashtra (19\%), Gujarat (11\%), Uttar Pradesh (8\%) and Haryana (6\%), (Sonawane et al., 2010). The nutrient content of pearlmillet compares very well with other cereals and millets. It has high protein content with slightly superior amino acid profile. Pearlmillet grain contains 13-14 per cent protein, 5-6 per cent fat, 74 per cent carbohydrate and 1-2 per cent minerals. It also contains higher amount of carotene, riboflavin (Vitamin B2) and niacin Vitamin B4 (Singh et al., 2009).

\section{Materials and Methods}

Field experiment was conducted during Zaid season 2015 at Crop Research Farm, Sam Higginbottom Institute of Agriculture, Technology and Sciences (Deemed-to-beUniversity) Allahabad.

The soil of the experimental area was sandy loam with moderately alkaline $\mathrm{pH}$; low in organic carbon $(0.32 \%)$ and available $\mathrm{N}$ (188.30 kg ha $\left.{ }^{-1}\right)$, available P (34.50 kg ha $\left.{ }^{-1}\right)$ and available $\mathrm{K}\left(87.00 \mathrm{~kg} \mathrm{ha}^{-1}\right)$ during zaid 2015 respectively. A recommended pearlmillet variety (Pro Agro 9444, Ganga kaveri 1044 and Pioneer 86 M 32) was chosen for the study.

The experiment was laid out in Randomized Block Design (RBD) with two factor different levels of Nitrogen and three improved Varieties with nine treatments combination on a plot size of $3 \times 3 \mathrm{~m}^{2}$. Before sowing, line were formed in the field as the spacing in treatments. Pearlmillet was sown in line and covered with the soil. Pearlmillet seeds were hand dibbled. The total quantity of nitrogen, phosphorus and potassium as per treatment in the form of Urea (46\%), single super phosphate (16\%) and MOP (60\%) respectively were applied below the seeds at the time of sowing.

Two split application are applied, one at basal and the second application at top dressing. All the agronomic practices were carried out uniformly to raised the crop. For taking data on yield and yield components on pearlmillet five plants were selected randomly in each plot.

\section{Results and Discussion}

\section{Plant height (cm)}

The observations for plant height are being presented in the table 1. A perusal of this table reveals that there was a steady increase in the plant height between the day's intervals. The significant influences were observed in plant height due to different treatments.

There was significant difference between the treatments and maximum plant height (175.30 $\mathrm{cm})$ was observed by the application of $\mathrm{T} 9$ i.e., V3 + 100:45:45 kg ha ${ }^{-1} \mathrm{NPK}$, whereas the lowest value $164.47 \mathrm{~cm}$ was observed in treatment T1 i.e., V1 + 80:45:45 $\mathrm{kg} \mathrm{ha}^{-1} \mathrm{NPK}$. Further, treatments, T3 i.e., V1 + 100:45:45kg $\mathrm{ha}^{-1}$ NPK, T6 i.e., V2 + 100:45:45kg ha ${ }^{-1}$ 
NPK, T7 i.e., V 3+ 80:45:45 $\mathrm{kg} \mathrm{ha}^{-1}$, T8 i.e., $\mathrm{V} 3+90: 45: 45 \mathrm{~kg} \mathrm{ha}^{-1} \mathrm{NPK}, \mathrm{T} 2$ i.e., V1 + 90:45:45 $\mathrm{kg} \mathrm{ha}^{-1}$ NPK and T4 i.e., V2+ $80: 45: 45 \mathrm{~kg} \mathrm{ha}^{-1} \mathrm{NPK}$ were found statistically at par with T9 i.e., V3 + 100:45:45 kg ha-1 NPK.

The probable reasons for recording higher stature of growth attributes viz., plant height, leaf area index, dry matter production and number of tillers $\mathrm{m}^{-2}$ was observed in different varieties due to increased levels of nitrogen with the application of $50 \mathrm{~kg} \mathrm{~N} \mathrm{ha}^{-1}$.

While all these parameters were at their lowest value with no nitrogen application in foxtail millet (Setaria italica L.) Naik et al., (1995) and Basavarajappa et al., (2002) were also observed similar finding in pearlmillet by AICRP Forage Crops (2006).

\section{Tillers plant ${ }^{-1}$}

The observations for tillers plant ${ }^{-1}$ are being presented in the table 1 . Perusal of this table reveals that there was a steady increase in the tillers plant ${ }^{-1}$ at all the day's intervals. The significant influences were observed in tillers plant $^{-1}$ due to different treatments.

The maximum tillers plant $^{-1}$ (3.67) was observed by the application of $\mathrm{T} 9$ i.e., $\mathrm{V} 3+$ 100:45:45 $\mathrm{kg} \mathrm{ha}^{-1} \mathrm{NPK}$, whereas the lowest value 2.60 was observed in treatment $\mathrm{T} 1$ i.e., $\mathrm{V} 1+80: 45: 45 \mathrm{~kg} \mathrm{ha}^{-1}$ NPK (Table 2).

Further, treatments, T3 i.e., V1 + 100:45:45kg $\mathrm{ha}^{-1}$ NPK was found statistically at par with T9 i.e., V3 + 100:45:45 $\mathrm{kg} \mathrm{ha}^{-1} \mathrm{NPK}$.

The probable reasons for recording higher stature of growth attributes viz., plant height, leaf area index, dry matter production and number of tillers $\mathrm{m}^{2}$ was observed in different varieties due to increased levels of nitrogen with the application of $50 \mathrm{~kg} \mathrm{~N} \mathrm{ha}^{-1}$.
While all these parameters were at their lowest value with no nitrogen application in foxtail millet (Setaria italica L.) Naik et al., (1995) and Basavarajappa et al., (2002) observed the similar finding in pearlmillet by AICRP Forage Crops (2006).

Experimental findings showed that the effect of nitrogen fertilization (30, 60 and $90 \mathrm{~kg} \mathrm{ha}^{-1}$ ) on growth and yield of pearlmillet and observed significant improvement in plant height, number of green leaves and number of tillers with 60 and $90 \mathrm{~kg}$ nitrogen over $30 \mathrm{~kg}$ $\mathrm{ha}^{-1}$. The results are in conformity with those of Chaudhari et al., (2002) Singh et al., (1991), and Babu et al., (1995).

\section{Number of ear plant ${ }^{-1}$}

The result revealed that there was significant difference between the treatments and maximum ear plant $^{-1}\left(2.37\right.$ plant $\left.^{-1}\right)$ was observed by the application of T9 i.e., V3 + 100:45:45 kg ha ${ }^{-1}$ NPK whereas the lowest value 1.37 plant $^{-1}$ was observed in treatment T1 i.e., V1 + 80:45:45 kg ha ${ }^{-1}$ NPK. However, treatment, T3 i.e., $\mathrm{V} 1+100: 45: 45 \mathrm{~kg} \mathrm{ha}^{-1}$ NPK, T6 i.e., V2 + 100:45:45kg ha ${ }^{-1} \mathrm{NPK}$ was found statistically at par with T9 i.e., V3 + 100:45:45 kg ha ${ }^{-1}$ NPK.

\section{Length of ear $(\mathrm{cm})$}

The result revealed that there was significant difference between the treatments and maximum length of ear $(27.52 \mathrm{~cm})$ was obtained in T9 i.e., V3 + 100:45:45 kg ha NPK whereas the lowest value $21.16 \mathrm{~cm}$ was observed in treatment T8 i.e., V3 + 90:45:45 $\mathrm{kg} \mathrm{ha}^{-1} \mathrm{NPK}$. 
Table.1 Response of hybrid and different levels of nitrogen on plant height of pearlmillet at different intervals

\begin{tabular}{|c|c|c|c|c|c|}
\hline \multicolumn{2}{|r|}{ Treatment } & \multicolumn{4}{|c|}{ Plant height (cm) } \\
\hline & & $\begin{array}{c}20 \\
\text { DAS }\end{array}$ & 40 DAS & 60 DAS & 80 DAS \\
\hline $\mathrm{T}_{1}$ & $\mathrm{~V}_{1}+80: 45: 45 \mathrm{~kg} \mathrm{ha}^{-1} \mathrm{NPK}$ & 34.80 & 101.67 & 161.70 & 164.47 \\
\hline $\mathrm{T}_{2}$ & $\mathrm{~V}_{1}+90: 45: 45 \mathrm{~kg} \mathrm{ha}^{-1} \mathrm{NPK}$ & 34.98 & 102.80 & 165.47 & 171.20 \\
\hline $\mathrm{T}_{3}$ & $\mathrm{~V}_{1}+100: 45: 45 \mathrm{~kg} \mathrm{ha}^{-1} \mathrm{NPK}$ & 39.47 & 109.10 & 168.40 & 173.60 \\
\hline $\mathrm{T}_{4}$ & $\mathrm{~V}_{2}+80: 45: 45 \mathrm{~kg} \mathrm{ha}^{-1} \mathrm{NPK}$ & 35.27 & 102.97 & 161.80 & 170.43 \\
\hline $\mathrm{T}_{5}$ & $\mathrm{~V}_{2}+90: 45: 45 \mathrm{~kg} \mathrm{ha}^{-1} \mathrm{NPK}$ & 35.37 & 106.30 & 166.43 & 167.67 \\
\hline $\mathrm{T}_{6}$ & $\mathrm{~V}_{2}+100: 45: 45 \mathrm{~kg} \mathrm{ha}^{-1} \mathrm{NPK}$ & 38.50 & 108.40 & 167.23 & 172.53 \\
\hline $\mathrm{T}_{7}$ & $\mathrm{~V}_{3}+80: 45: 45 \mathrm{~kg} \mathrm{ha}^{-1} \mathrm{NPK}$ & 36.23 & 106.27 & 165.30 & 171.53 \\
\hline $\mathrm{T}_{8}$ & $\mathrm{~V}_{3}+90: 45: 45 \mathrm{~kg} \mathrm{ha}^{-1} \mathrm{NPK}$ & 36.40 & 103.37 & 168.40 & 171.23 \\
\hline $\mathrm{T}_{9}$ & $\mathrm{~V}_{3}+100: 45: 45 \mathrm{~kg} \mathrm{ha}^{-1} \mathrm{NPK}$ & 46.17 & 110.60 & 169.30 & 175.30 \\
\hline & F- test & NS & $\mathrm{S}$ & $\mathrm{S}$ & $\mathrm{S}$ \\
\hline & S. Ed. $( \pm)$ & 5.343 & 2.697 & 2.285 & 2.781 \\
\hline & C. D. $(P=0.05)$ & - & 5.718 & 4.844 & 5.895 \\
\hline
\end{tabular}

Table.2 Response of hybrid and different levels of nitrogen on tillers plant ${ }^{-1}$ of pearlmillet at different intervals

\begin{tabular}{cccrrr}
\hline & Treatment & \multicolumn{4}{c}{ Tillers plant $^{-1}$} \\
& & 20 & 40 DAS & 60 DAS & 80 DAS \\
\hline $\mathrm{T}_{1}$ & $\mathrm{~V}_{1}+80: 45: 45 \mathrm{~kg} \mathrm{ha}^{-1} \mathrm{NPK}$ & 0.60 & 1.47 & 2.73 & 2.60 \\
$\mathrm{~T}_{2}$ & $\mathrm{~V}_{1}+90: 45: 45 \mathrm{~kg} \mathrm{ha}^{-1} \mathrm{NPK}$ & 0.80 & 1.87 & 2.87 & 2.73 \\
$\mathrm{~T}_{3}$ & $\mathrm{~V}_{1}+100: 45: 45 \mathrm{~kg} \mathrm{ha}^{-1} \mathrm{NPK}$ & 1.00 & 2.20 & 3.60 & 3.33 \\
$\mathrm{~T}_{4}$ & $\mathrm{~V}_{2}+80: 45: 45 \mathrm{~kg} \mathrm{ha}^{-1} \mathrm{NPK}$ & 0.67 & 1.87 & 2.73 & 2.60 \\
$\mathrm{~T}_{5}$ & $\mathrm{~V}_{2}+90: 45: 45 \mathrm{~kg} \mathrm{ha}^{-1} \mathrm{NPK}$ & 0.80 & 1.93 & 2.87 & 2.87 \\
$\mathrm{~T}_{6}$ & $\mathrm{~V}_{2}+100: 45: 45 \mathrm{~kg} \mathrm{ha}^{-1} \mathrm{NPK}$ & 0.87 & 2.07 & 3.13 & 3.13 \\
$\mathrm{~T}_{7}$ & $\mathrm{~V}_{3}+80: 45: 45 \mathrm{~kg} \mathrm{ha}^{-1} \mathrm{NPK}$ & 0.67 & 1.67 & 2.87 & 2.87 \\
$\mathrm{~T}_{8}$ & $\mathrm{~V}_{3}+90: 45: 45 \mathrm{~kg} \mathrm{ha}^{-1} \mathrm{NPK}$ & 0.67 & 1.80 & 2.93 & 3.13 \\
$\mathrm{~T}_{9}$ & $\mathrm{~V}_{3}+100: 45: 45 \mathrm{~kg} \mathrm{ha}^{-1} \mathrm{NPK}$ & 1.13 & 2.60 & 3.78 & 3.67 \\
\hline & F- test & $\mathrm{NS}$ & $\mathrm{S}$ & $\mathrm{S}$ & $\mathrm{S}$ \\
& S. Ed. $( \pm)$ & 0.239 & 0.208 & 0.270 & 0.245 \\
& & - & 0.442 & 0.574 & 0.520 \\
\hline
\end{tabular}


Table.3 Response of hybrid and different levels of nitrogen on Dry weight of pearlmillet at different intervals

\begin{tabular}{|c|c|c|c|c|c|c|}
\hline & \multirow[t]{2}{*}{ Treatment } & & \multicolumn{4}{|c|}{ Dry weight (g plant ${ }^{-1}$ ) } \\
\hline & & & $\begin{array}{c}20 \\
\text { DAS }\end{array}$ & 40 DAS & 60 DAS & 80 DAS \\
\hline $\mathrm{T}_{1}$ & $\mathrm{~V}_{1}+80: 45: 45 \mathrm{~kg} \mathrm{ha}^{-1} \mathrm{NPK}$ & 0.33 & 7.03 & 16.13 & 40.80 & \\
\hline $\mathrm{T}_{2}$ & $\mathrm{~V}_{1}+90: 45: 45 \mathrm{~kg} \mathrm{ha}^{-1} \mathrm{NPK}$ & 0.37 & 7.78 & 16.54 & 45.37 & \\
\hline $\mathrm{T}_{3}$ & $\mathrm{~V}_{1}+100: 45: 45 \mathrm{~kg} \mathrm{ha}^{-1} \mathrm{NPK}$ & 0.44 & 8.52 & 18.33 & 47.32 & \\
\hline $\mathrm{T}_{4}$ & $\mathrm{~V}_{2}+80: 45: 45 \mathrm{~kg} \mathrm{ha}^{-1} \mathrm{NPK}$ & 0.35 & 7.55 & 16.72 & 42.32 & \\
\hline $\mathrm{T}_{5}$ & $\mathrm{~V}_{2}+90: 45: 45 \mathrm{~kg} \mathrm{ha}^{-1} \mathrm{NPK}$ & 0.38 & 7.36 & 16.93 & 42.15 & \\
\hline $\mathrm{T}_{6}$ & $\mathrm{~V}_{2}+100: 45: 45 \mathrm{~kg} \mathrm{ha}^{-1} \mathrm{NPK}$ & 0.39 & 8.22 & 17.62 & 46.27 & \\
\hline $\mathrm{T}_{7}$ & $\mathrm{~V}_{3}+80: 45: 45 \mathrm{~kg} \mathrm{ha}^{-1} \mathrm{NPK}$ & 0.37 & 7.53 & 16.76 & 42.01 & \\
\hline $\mathrm{T}_{8}$ & $\mathrm{~V}_{3}+90: 45: 45 \mathrm{~kg} \mathrm{ha}^{-1} \mathrm{NPK}$ & 0.34 & 7.25 & 16.69 & 42.57 & \\
\hline $\mathrm{T}_{9}$ & $\mathrm{~V}_{3}+100: 45: 45 \mathrm{~kg} \mathrm{ha}^{-1} \mathrm{NPK}$ & 0.45 & 9.03 & 18.50 & 47.73 & \\
\hline & F- test & NS & $\mathrm{S}$ & $\mathrm{S}$ & $\mathrm{S}$ & \\
\hline & S. Ed. $( \pm)$ & 0.044 & 0.424 & 0.722 & 1.862 & \\
\hline & C. D. $(P=0.05)$ & - & 0.900 & 1.532 & 3.948 & \\
\hline
\end{tabular}

Table.4 Response of hybrid and different levels of nitrogen on Crop Growth Rate of pearlmillet at different intervals

\begin{tabular}{|c|c|c|c|c|c|c|}
\hline & \multirow[t]{3}{*}{ Treatment } & & \multicolumn{4}{|c|}{ Crop Growth Rate $\left(\mathrm{g} \mathrm{m}^{-2}\right.$ day $\left.^{-1}\right)$} \\
\hline & & & 0-20 & $20-40$ & $40-60$ & $60-80$ \\
\hline & & & DAS & DAS & DAS & DAS \\
\hline $\mathrm{T}_{1}$ & $\mathrm{~V}_{1}+80: 45: 45 \mathrm{~kg} \mathrm{ha}^{-1} \mathrm{NPK}$ & 0.016 & 0.34 & 0.44 & 1.30 & \\
\hline $\mathrm{T}_{2}$ & $\mathrm{~V}_{1}+90: 45: 45 \mathrm{~kg} \mathrm{ha}^{-1} \mathrm{NPK}$ & 0.018 & 0.33 & 0.44 & 1.36 & \\
\hline $\mathrm{T}_{3}$ & $\mathrm{~V}_{1}+100: 45: 45 \mathrm{~kg} \mathrm{ha}^{-1} \mathrm{NPK}$ & 0.021 & 0.40 & 0.51 & 1.42 & \\
\hline $\mathrm{T}_{4}$ & $\mathrm{~V}_{2}+80: 45: 45 \mathrm{~kg} \mathrm{ha}^{-1} \mathrm{NPK}$ & 0.017 & 0.37 & 0.43 & 1.37 & \\
\hline $\mathrm{T}_{5}$ & $\mathrm{~V}_{2}+90: 45: 45 \mathrm{~kg} \mathrm{ha}^{-1} \mathrm{NPK}$ & 0.018 & 0.36 & 0.46 & 1.38 & \\
\hline $\mathrm{T}_{6}$ & $\mathrm{~V}_{2}+100: 45: 45 \mathrm{~kg} \mathrm{ha}^{-1} \mathrm{NPK}$ & 0.019 & 0.37 & 0.50 & 1.39 & \\
\hline $\mathrm{T}_{7}$ & $\mathrm{~V}_{3}+80: 45: 45 \mathrm{~kg} \mathrm{ha}^{-1} \mathrm{NPK}$ & 0.018 & 0.35 & 0.45 & 1.30 & \\
\hline $\mathrm{T}_{8}$ & $\mathrm{~V}_{3}+90: 45: 45 \mathrm{~kg} \mathrm{ha}^{-1} \mathrm{NPK}$ & 0.016 & 0.34 & 0.39 & 1.37 & \\
\hline $\mathrm{T}_{9}$ & $\mathrm{~V}_{3}+100: 45: 45 \mathrm{~kg} \mathrm{ha}^{-1} \mathrm{NPK}$ & 0.022 & 0.40 & 0.53 & 1.53 & \\
\hline & F- test & NS & $\mathrm{S}$ & NS & NS & \\
\hline & S. Ed. $( \pm)$ & 0.002 & 0.021 & 0.06 & 0.114 & \\
\hline & C. D. $(P=0.05)$ & - & 0.045 & - & - & \\
\hline
\end{tabular}


Table.5 Response of hybrid and different levels of nitrogen on Relative Growth Rate of pearlmillet at different intervals

\begin{tabular}{cccccc}
\hline & & Treatment & \multicolumn{3}{c}{ Relative Growth Rate $\left(\mathbf{g ~ g}^{-1}\right.$ day $\left.^{-1}\right)$} \\
& & $\mathbf{0 - 2 0}$ & $\mathbf{2 0 - 4 0}$ & $\mathbf{4 0 - 6 0}$ & $\mathbf{6 0 - 8 0}$ \\
& & DAS & DAS & DAS & DAS \\
\hline $\mathrm{T}_{1}$ & $\mathrm{~V}_{1}+80: 45: 45 \mathrm{~kg} \mathrm{ha}^{-1} \mathrm{NPK}$ & 0.05 & 0.15 & 0.035 & 0.045 \\
$\mathrm{~T}_{2}$ & $\mathrm{~V}_{1}+90: 45: 45 \mathrm{~kg} \mathrm{ha}^{-1} \mathrm{NPK}$ & 0.04 & 0.15 & 0.037 & 0.047 \\
$\mathrm{~T}_{3}$ & $\mathrm{~V}_{1}+100: 45: 45 \mathrm{~kg} \mathrm{ha}^{-1} \mathrm{NPK}$ & 0.04 & 0.16 & 0.044 & 0.048 \\
$\mathrm{~T}_{4}$ & $\mathrm{~V}_{2}+80: 45: 45 \mathrm{~kg} \mathrm{ha}^{-1} \mathrm{NPK}$ & 0.05 & 0.15 & 0.035 & 0.047 \\
$\mathrm{~T}_{5}$ & $\mathrm{~V}_{2}+90: 45: 45 \mathrm{~kg} \mathrm{ha}^{-1} \mathrm{NPK}$ & 0.03 & 0.14 & 0.039 & 0.047 \\
$\mathrm{~T}_{6}$ & $\mathrm{~V}_{2}+100: 45: 45 \mathrm{~kg} \mathrm{ha}^{-1} \mathrm{NPK}$ & 0.04 & 0.15 & 0.043 & 0.047 \\
$\mathrm{~T}_{7}$ & $\mathrm{~V}_{3}+80: 45: 45 \mathrm{~kg} \mathrm{ha}^{-1} \mathrm{NPK}$ & 0.03 & 0.15 & 0.039 & 0.046 \\
$\mathrm{~T}_{8}$ & $\mathrm{~V}_{3}+90: 45: 45 \mathrm{~kg} \mathrm{ha}^{-1} \mathrm{NPK}$ & 0.05 & 0.15 & 0.032 & 0.047 \\
$\mathrm{~T}_{9}$ & $\mathrm{~V}_{3}+100: 45: 45 \mathrm{~kg} \mathrm{ha}^{-1} \mathrm{NPK}$ & 0.05 & 0.16 & 0.045 & 0.052 \\
& & & & & \\
\hline & $\mathrm{F}-$ test & $\mathrm{NS}$ & $\mathrm{S}$ & $\mathrm{NS}$ & $\mathrm{NS}$ \\
& $\mathrm{S}$. Ed. $( \pm)$ & 0.007 & 0.005 & 0.004 & 0.002 \\
& C. D. $(\mathrm{P}=0.05)$ & - & 0.011 & - & - \\
\hline
\end{tabular}

Table.6 Response of hybrid and different levels of nitrogen on protein content of pearlmillet

\begin{tabular}{ccc}
\hline & Treatment & $\begin{array}{c}\text { Protein content } \\
(\boldsymbol{\%})\end{array}$ \\
\hline $\mathrm{T}_{1}$ & $\mathrm{~V}_{1}+80: 45: 45 \mathrm{~kg} \mathrm{ha}^{-1} \mathrm{NPK}$ & 9.75 \\
$\mathrm{~T}_{2}$ & $\mathrm{~V}_{1}+90: 45: 45 \mathrm{~kg} \mathrm{ha}^{-1} \mathrm{NPK}$ & 11.43 \\
$\mathrm{~T}_{3}$ & $\mathrm{~V}_{1}+100: 45: 45 \mathrm{~kg} \mathrm{ha}^{-1} \mathrm{NPK}$ & 12.78 \\
$\mathrm{~T}_{4}$ & $\mathrm{~V}_{2}+80: 45: 45 \mathrm{~kg} \mathrm{ha}^{-1} \mathrm{NPK}$ & 11.16 \\
$\mathrm{~T}_{5}$ & $\mathrm{~V}_{2}+90: 45: 45 \mathrm{~kg} \mathrm{ha}^{-1} \mathrm{NPK}$ & 9.48 \\
$\mathrm{~T}_{6}$ & $\mathrm{~V}_{2}+100: 45: 45 \mathrm{~kg} \mathrm{ha}^{-1} \mathrm{NPK}$ & 12.10 \\
$\mathrm{~T}_{7}$ & $\mathrm{~V}_{3}+80: 45: 45 \mathrm{~kg} \mathrm{ha}^{-1} \mathrm{NPK}$ & 10.78 \\
$\mathrm{~T}_{8}$ & $\mathrm{~V}_{3}+90: 45: 45 \mathrm{~kg} \mathrm{ha}^{-1} \mathrm{NPK}$ & 10.02 \\
$\mathrm{~T}_{9}$ & $\mathrm{~V}_{3}+100: 45: 45 \mathrm{~kg} \mathrm{ha}^{-1} \mathrm{NPK}$ & 13.43 \\
& & $\mathrm{~S}$ \\
\hline & $\mathrm{F}-\mathrm{test}$ & 0.001 \\
& $\mathrm{~S}$. Ed. $( \pm)$ & 0.003 \\
\hline
\end{tabular}


Table.7 Response of hybrid and different levels of nitrogen on yield and yield attributes of pearlmillet

\begin{tabular}{|c|c|c|c|c|c|c|c|c|}
\hline & Treatment & $\begin{array}{l}\text { Ear } \\
\text { Plant }^{-1}\end{array}$ & $\begin{array}{l}\text { Length } \\
\text { of Ear }\end{array}$ & $\begin{array}{l}\text { No of } \\
\text { Grains per } \\
\text { Ear }\end{array}$ & $\begin{array}{l}\text { Test } \\
\text { weight } \\
\text { (gm) }\end{array}$ & $\begin{array}{l}\text { Grain } \\
\text { yield } \\
\text { (t ha } \\
\text { 1) }\end{array}$ & $\begin{array}{l}\text { Stover } \\
\text { yield } \\
\left(\mathrm{t} \mathrm{ha}^{-1}\right)\end{array}$ & $\begin{array}{l}\text { Harvest } \\
\text { index }(\%)\end{array}$ \\
\hline $\mathrm{T}_{1}$ & $\mathrm{~V}_{1}+80: 45: 45 \mathrm{~kg} \mathrm{ha}^{-1} \mathrm{NPK}$ & 1.37 & 22.77 & 2132.73 & 8.68 & 2.47 & 4.62 & 19.64 \\
\hline \multirow{3}{*}{$\begin{array}{l}\mathrm{T}_{2} \\
\mathrm{~T}_{3}\end{array}$} & $\mathrm{~V}_{1}+90: 45: 45 \mathrm{~kg} \mathrm{ha}^{-1} \mathrm{NPK}$ & 1.74 & 21.43 & 2062.00 & 8.25 & 2.78 & 5.33 & 23.03 \\
\hline & $\mathrm{V}_{1}+100: 45: 45 \mathrm{~kg} \mathrm{ha} \mathrm{ha}^{-1}$ & & & & & & & \\
\hline & NPK & 2.03 & 24.62 & 2256.26 & 9.23 & 3.14 & 6.45 & 25.01 \\
\hline $\mathrm{T}_{4}$ & $\mathrm{~V}_{2}+80: 45: 45 \mathrm{~kg} \mathrm{ha}^{-1} \mathrm{NPK}$ & 1.69 & 22.94 & 1963.00 & 8.12 & 2.76 & 5.05 & 19.01 \\
\hline \multirow{3}{*}{$\begin{array}{l}\mathrm{T}_{5} \\
\mathrm{~T}_{6}\end{array}$} & $\mathrm{~V}_{2}+90: 45: 45 \mathrm{~kg} \mathrm{ha}^{-1} \mathrm{NPK}$ & 1.44 & 22.28 & 1928.88 & 8.04 & 2.47 & 4.95 & 20.00 \\
\hline & $\mathrm{V}_{2}+100: 45: 45 \mathrm{~kg} \mathrm{ha} \mathrm{ha}^{-1}$ & & & & & & & \\
\hline & NPK & 2.01 & 24.06 & 2166.92 & 9.18 & 3.02 & 6.26 & 23.68 \\
\hline $\mathrm{T}_{7}$ & $\mathrm{~V}_{3}+80: 45: 45 \mathrm{~kg} \mathrm{ha}^{-1} \mathrm{NPK}$ & 1.63 & 22.44 & 2070.67 & 8.39 & 2.53 & 5.93 & 21.37 \\
\hline \multirow{6}{*}{$\begin{array}{l}\mathrm{T}_{8} \\
\mathrm{~T}_{9}\end{array}$} & $\mathrm{~V}_{3}+90: 45: 45 \mathrm{~kg} \mathrm{ha}^{-1} \mathrm{NPK}$ & 1.74 & 21.16 & 2138.80 & 8.81 & 3.01 & 5.44 & 22.85 \\
\hline & $\mathrm{V}_{3}+100: 45: 45 \mathrm{~kg} \mathrm{ha}^{-1}$ & & & & & & & \\
\hline & NPK & 2.37 & 27.52 & 2406.49 & 10.29 & 3.72 & 6.98 & 25.07 \\
\hline & F-test & $\mathrm{S}$ & $\mathrm{S}$ & $\mathrm{S}$ & $\mathrm{S}$ & $\mathrm{S}$ & $\mathrm{S}$ & $\mathrm{S}$ \\
\hline & S. Ed. $( \pm)$ & 0.229 & 1.08 & 84.91 & 0.589 & 0.350 & 0.608 & 1.790 \\
\hline & C. D. $(P=0.05)$ & 0.486 & 2.289 & 180.02 & 1.249 & 0.743 & 1.290 & 3.795 \\
\hline
\end{tabular}




\section{Number of grains ear ${ }^{-1}$}

The result revealed that there was significant difference between the treatments and maximum number of grains (2406.49 $\mathrm{ear}^{-1}$ ) was observed by the application ofV3 + 100:45:45 kg ha ${ }^{-1}$ NPK i.e., T9, whereas the lowest value $1928.88 \mathrm{ear}^{-1}$ was observed in treatment T5 i.e., $\mathrm{V} 2+$ 90:45:45 kg ha ${ }^{-1} \mathrm{NPK}$. However, treatment, T3 i.e., V1 + 100:45:45 $\mathrm{kg} \mathrm{ha}^{-1} \mathrm{NPK}$, was found statistically at par with T9 i.e., $\mathrm{V} 3+100: 45: 45 \mathrm{~kg} \mathrm{ha}^{-1}$ NPK.

\section{Test weight (g)}

The result revealed that there was significant difference between the treatments and maximum test weight $(10.29 \mathrm{~g})$ was observed by the application of T9 i.e., V3 + 100:45:45 $\mathrm{kg} \mathrm{ha}^{-1}$ NPK. Whereas the lowest value $8.04 \mathrm{~g}$ was observed in treatment T5 i.e., $\mathrm{V} 2+$ 90:45:45 kg ha ${ }^{-1}$ NPK. However, treatment, T3 i.e., V1 + 100:45:45 $\mathrm{kg} \mathrm{ha}^{-1} \mathrm{NPK}$, T6 i.e., $\mathrm{V} 2+100: 45: 45 \mathrm{~kg} \mathrm{ha}^{-1}$ NPK was found statistically at par with $\mathrm{T} 9$ i.e., $\mathrm{V} 3+$ 100:45:45 kg ha ${ }^{-1}$ NPK.

\section{Grain yield (t ha-1)}

The result revealed that there was significant difference between different treatments and maximum grain yield $\left(3.72 \mathrm{t} \mathrm{ha}^{-1}\right)$ was observed by the application in T9 i.e., V3 + 100:45:45 kg ha-1 NPK, whereas the lowest value $2.47 \mathrm{t} \mathrm{ha}^{-1}$ was observed in treatment $\mathrm{T} 5$ i.e., $\mathrm{V} 2+90: 45: 45 \mathrm{~kg} \mathrm{ha}^{-1} \mathrm{NPK}$ and T1 i.e., $\mathrm{V} 1+80: 45: 45 \mathrm{~kg} \mathrm{ha}^{-1}$ NPK. However, treatment, T3 i.e., $\mathrm{V} 1+100: 45: 45 \mathrm{~kg} \mathrm{ha}^{-1}$ NPK, T6 i.e., V2 + 100:45:45 $\mathrm{kg} \mathrm{ha}^{-1} \mathrm{NPK}$ was found statistically at par with T9 i.e., V3 $+100: 45: 45 \mathrm{~kg} \mathrm{ha}^{-1}$ NPK.

\section{Straw yield $\left(\mathrm{t} \mathrm{ha}{ }^{-1}\right)$}

The result revealed that there was significant difference between different treatments and maximum straw yield $\left(6.98 \mathrm{t} \mathrm{ha}^{-1}\right)$ was observed by the application in T9 i.e., V3 + 100:45:45 $\mathrm{kg} \mathrm{ha}^{-1}$ NPK, whereas the lowest value $4.62 \mathrm{t} \mathrm{ha}^{-1}$ was observed in treatment $\mathrm{T} 1$ i.e., $\mathrm{V} 1+80: 45: 45 \mathrm{~kg} \mathrm{ha}^{-1}$ NPK. However, treatment, T3 i.e., $\mathrm{V} 1+100: 45: 45 \mathrm{~kg} \mathrm{ha}^{-1}$ NPK, T6 i.e., V2 + 100:45:45 $\mathrm{kg} \mathrm{ha}^{-1}$ NPK was found statistically at par with T9 i.e., V3 $+100: 45: 45 \mathrm{~kg} \mathrm{ha}^{-1}$ NPK.

\section{Harvest index (\%)}

The result revealed that there was significant difference between the treatments and maximum harvest index $(25.07 \%)$ was observed by the application in T9 i.e., V3 + 100:45:45 $\mathrm{kg} \mathrm{ha}^{-1} \mathrm{NPK}$, whereas the lowest value $19.01 \%$ in T4 i.e., V 2+ 80:45:45 kg ha1 NPK N1. However, treatment, T3 i.e., V1 + 100:45:45 $\mathrm{kg} \mathrm{ha}^{-1}$ NPK, T6 i.e., V2 + 100:45:45 $\mathrm{kg} \mathrm{ha}^{-1}$ NPK, T2 i.e., V1 + 90:45:45kg ha- 1NPK, T8 i.e., V3 + 90:45:45 $\mathrm{kg} \mathrm{ha}^{-1} \mathrm{NPK}$ and T7 i.e., V 3+ 80:45:45 kg ha ${ }^{1}$ NPK was found statistically at par with T9 i.e., V3 + 100:45:45 $\mathrm{kg} \mathrm{ha}^{-1}$ NPK. Saini and Negi (1996), Munirathnam et al., (2006), observed that the highest harvest index was recorded in different varieties due to increased levels of nitrogen with $25 \mathrm{~kg} \mathrm{~N}^{-1}$ which was however, comparable with no nitrogen application in foxtail millet (Setaria italica L.). The probable reasons for recording significantly increase in grain and straw yields were observed with increase in nitrogen levels from 0 to $50 \mathrm{~kg} \mathrm{~N} \mathrm{ha}^{-1}$. The lowest grain and straw yields were recorded with no nitrogen application in foxtail millet (Setaria italica L.) Saini and Negi (1996), Munirathnam et al., (2006) (Table 7).

Among the response of different hybrids to levels of nitrogen, treatment $\mathrm{T} 9$ i.e., $\mathrm{V} 3+$ 100:45:45 kg ha ${ }^{-1}$ NPK, recorded maximum plant height $(175.30 \mathrm{~cm})$, number of tillers plant $^{-1}$ (3.67), dry weight (47.73g), crop growth rate $\left(1.53 \mathrm{~g} \mathrm{~m}-2\right.$ day $\left.^{-1}\right)$, relative growth rate $\left(0.052 \mathrm{~g} \mathrm{~g}^{-1} \mathrm{day}^{-1}\right)$, number of ear 
plant $^{-1} 2.37$, length of ear $(27.52 \mathrm{~cm})$, number of Grains ear ${ }^{-1}$ (2406.49), test weight $(10.29 \mathrm{~g})$, grain yield $\left(3.72 \mathrm{t} \mathrm{ha}^{-1}\right)$, straw yield $\left(6.98 \mathrm{t} \mathrm{ha}^{-1}\right)$, protein content $(13.43 \%)$ and harvest index (36.15\%). Whereas the lowest value in terms of plant height $(164.47 \mathrm{~cm})$, number of tillers $\left(2.60\right.$ plant $\left.^{-1}\right)$, dry weight $(40.80 \mathrm{~g})$ (Table 3), crop growth rate $(1.30 \mathrm{~g}$ $\mathrm{m}^{-2}$ day $^{-1}$ ) (Table 4), relative growth rate $\left(0.045 \mathrm{~g} \mathrm{~g}^{-1} \mathrm{day}^{-1}\right)$ (Table 5), number of ear $\left(1.37\right.$ plant $\left.^{-1}\right)$, grain yield $\left(2.47 \mathrm{t} \mathrm{ha}^{-1}\right)$ and straw yield $\left(4.62 \mathrm{t} \mathrm{ha}^{-1}\right)$ was observed in the treatment $\mathrm{T} 1$ i.e., $\mathrm{V} 1+80: 45: 45 \mathrm{~kg} \mathrm{ha}^{-1}$ NPK). Further, ear length of $(21.16 \mathrm{~cm})$ was observed in the treatment $\mathrm{T} 8$ i.e., $\mathrm{V} 3+$ 90:45:45 $\left.\mathrm{kg} \mathrm{ha}^{-1} \mathrm{NPK}\right)$. In terms of number of grains $\left(2406.49\right.$ ear $\left.^{-1}\right)$, test weight $(8.04 \mathrm{~g})$ was recorded in the treatment T5 i.e., V2 + 90:45:45 kg ha ${ }^{-1}$ NPK). Further, protein content $(9.48 \%$ ) (Table 6 ) was observed in the treatment T5 i.e., $\mathrm{V} 2$ + 90:45:45 $\left.\mathrm{kg} \mathrm{ha}^{-1} \mathrm{NPK}\right)$ and harvest index (19.01\%) respectively in the treatment T4 i.e., $\mathrm{V} 2+80: 45: 45 \mathrm{~kg} \mathrm{ha}^{-1}$ NPK).

\section{References}

AICRP-Forage Crops, 2006. Effect of nitrogenlevels on promising entries of pearlmillet. All India Co-ordinated Research Project on forage crops. IGFRI Jhansi, Annual Report Kharif- 2005: 161177.

Anonymous. 2010-2011. Annual Report All India Co-ordinated Pearlmillet Improvement Project pp.141-142.

Babu, R.S., Patil, G.T.S. and Prabhakar, A.S. 1995. Effect of stage of cutting, nitrogen and phosphorus level on forage pearlmillet
(Pennisetum glaucum L.) Forage Res., 20: $225-231$.

Basavarajappa, et al. 2002. Response of safflower to bio-fertilizers with nitrogen levels on growth and seed yield.

Chaudhari, A.C., Meena, N.L. and Jat, R.L. 2002. Effect of nitrogen and moisture conservation practices on growth and yield of rainfed pearlmillet. Annals Agri. Res., 23(2): 223-225.

Munirathnam, P., Reddy, A., Sambasiva and Sawadhkar, S.M. 2006. Evaluation of foxtail millet varieties under low fertility conditions. Agri. Sci. Digest, 26(3): 197199.

Naik, B., Linge Gowda, T.B.K., Thimme Gowda, S. and Sridhara, S. 1995. Effect of integrated nutrient management on growth and grain yield of foxtail millet (Setaria italica L. Beauv.) under rainfed conditions on Alfisols of sub-tropical India. Fertilizer News, 40(3): 55-57.

Saini, J.P. and Negi, S.C. 1996. Response of foxtail millet (Setaria italica) genotypes to nitrogen fertilization under dry-temperate conditions. Indian J. Agron., 41(2): 261264.

Singh, J., Randhawa, N.S. and Sidhu, M.S. 1991. Uptake of nitrogen and optimum levels for different genotypes of pearl millet. J. Res. PAU, Ludhiana, 28: 174178.

Sonawane, P.D., Wadile, S.C., Girase, P.P., Chitodkar, S.S. and Sonawane, D.A. 2010. Response of summer pearlmillet (Pennisetum glauchum L.) to depth and time of irrigation. Scheduling. Int. J. Agri. Sci., 6(1): 283-285.

\section{How to cite this article:}

Neha, Gautam Ghosh, Preeti Choudhary and Shobha Kumari. 2017. Effect of Different Nitrogen Levels and Varietal Performance on Growth and Yield of Summer Pearlmillet. Int.J.Curr.Microbiol.App.Sci. 6(6): 861-869. doi: https://doi.org/10.20546/ijcmas.2017.606.101 